\title{
Requirement Analysis of High-End Manufacturing Equipment with a Focus on the High-End Equipment Manufacturing Industry
}

\author{
Zhang Jun, Lu Bingheng
}

State Key Laboratory for Manufacturing Systems Engineering, Xi'an Jiaotong University, Xi'an 710049, China

\begin{abstract}
The continual development of high-end manufacturing equipment is essential for the development of the high-end equipment manufacturing industry. By examining the development trends of aeronautic equipment, aerospace equipment, rail transportation equipment, ocean engineering equipment, and intelligent manufacturing equipment during the 13th Five-Year Plan, these authors analyze the new demands and future priorities of high-end manufacturing equipment. Starting from the current situation of the manufacturing equipment enterprises in China, this paper puts forward suggestions to promote the sound development of the high-end equipment manufacturing industry.
\end{abstract}

Keywords: high-end equipment manufacturing industry; high-end manufacturing equipment; machine tool; basic manufacturing equipment

\section{Introduction}

The high-end equipment manufacturing industry, which belongs to the high-end manufacturing sector, is one of the seven strategic emerging industries during the 12th Five-Year Plan and the basic area of focus of China's strategy with high technology and added value. The development of the high-end equipment manufacturing industry has a great strategic significance in promoting the optimization and upgrading of China's industrial structure, and enhancing the core competitiveness of the manufacturing industry. According to the focus fields and directions of the Decision of the State Council on Accelerating the Cultivation and Development of Strategic Emerging Industries, the key directions, at the present stage, of the development of the high-end equipment manufacturing industry focus on aeronautic equipment, aerospace equipment, rail transportation equipment, ocean engineering equipment, and intelligent manufacturing equipment.
"Sharp tools make good work," and the manufacturing of high-end equipment needs the support of high-end machine tools and other basic equipment for casting, forging, welding, etc. The high-end manufacturing equipment, whose full development is an important foundation for national defense security, is essential for implementing various major scientific and technological projects such as integrated circuit manufacturing, large aircraft, nuclear power, inertial confinement fusion, earth observation, and aeroengine and industrial gas turbine engineering. If we lack high-end manufacturing and equipment technologies, strictly blocked by foreign countries in general, it will seriously restrict China's construction of national defense capability and the development of strategic products. In recent years, Europe and the United States have successively issued a number of action plans in the field of high-end manufacturing to promote the development of the equipment manufacturing industry. The United States launched the Advanced Manufacturing Partnership (AMP) program; Germany introduced the "Industry 4.0 " project; The

Received date: April 25, 2017; Revised date: May 31, 2017

Corresponding author: Lu Bingheng, Xi'an Jiaotong University, Professor, Chinese Academy of Engineering, Academician. Major research fields include high-end manufacturing equipment and additive manufacturing equipment. E-mail: bhlu@xjtu.edu.cn

Funding program: CAE Advisory Project "High-end Equipment Manufacturing and Its Requirement for High-end Machine tool" (2015-XY-37)

Chinese version: Strategic Study of CAE 2017, 19 (3): 136-141

Cited item: Zhang Jun et al. Requirement Analysis of High-End Manufacturing Equipment with a Focus on the High-End Equipment Manufacturing Industry. Strategic Study of CAE, https://doi.org/10.15302/J-SSCAE-2017.03.019 
United Kingdom established seven advanced manufacturing research centers and launched the High Value Manufacturing Strategy; The EU launched the intelligent manufacturing system 2020 plan; China began the implementation of the "Made in China 2025" plan in 2015. These plans have all been deployed in relation to the high-end manufacturing equipment.

High-end manufacturing equipment is the foundation of the realization of high-end equipment manufacturing. Therefore, whether high-end equipment could be made domestically, for a large extent, depends on the technical level of China's high-end manufacturing equipment. With the rapid development of China in the fields of aviation, aerospace, rail transportation, and ocean engineering, the requirement for high-end manufacturing equipment has increased with the equipment in these fields gradually developing towards a high, sophisticated, and advanced level. Starting from these four main areas for the development of the high-end equipment manufacturing industry in our country, we analyze the development trend of the 13th Five-Year Plan and the required high-end manufacturing equipment in depth, and propose suggestions for strategic development.

\section{Development of high-end equipment and requirement analysis of high-end manufacturing equipment}

\subsection{Aeronautic equipment}

It is estimated that the economic rationality of the aviation industry's survival and development will be significantly increased in the next 10 years. The intensity of key technology research and development will continue to increase, and there will be new changes in the industrial pattern. The delivery of mainline aircraft will account for more than $5 \%$ of the domestic market. The test flight of the C919 passenger aircraft opened the era of domestic large aircraft. The delivery of the turboprop regional aircraft will account for about $20 \%$ of the global market share. The delivery of general aircraft and helicopters will account for $20 \%$ and $10 \%$ respectively of the global market share. With the industrial development of aeroengine, some products began to occupy the domestic aircraft market. Airborne equipment and systems and auxiliary products have been manufactured with an annual output value of 50 billion yuan; Three levels, "systems, equipment and devices," of aviation equipment and supporting systems have been initially established; the long-term, stable, high-quality, and reliable supporting system and a complete industrial chain of aviation materials and components are being established.

With the continuous implementation of projects for key guarantee construction in the aviation industry over the years, the digital manufacturing technology has been widely adopted in aviation manufacturing. The three-dimensional digital model is preliminarily used to transmit information in the process of de- signing, manufacturing, testing, and assembling, and it, replacing the two-dimensional plane drawings, is a basis for manufacturing and testing. Owing to the overall lack of domestic manufacturing equipment, aeronautic manufacturing enterprises rely mainly on the procurement of imported manufacturing equipment and are restricted by the Western countries in many ways. According to the development trend of China's aviation industry and the development and production of aviation products, further improving of manufacturing equipment is imperative. The key manufacturing equipment in the aeronautical field should adapt to the development of new materials, new structures, and new processes in the research of next-generation aviation products, and realize the digitization, flexible integration, and intelligent control in the whole process of production. The characteristics of aeronautic products, changing rapidly from small batch to mass production, low costs, and high productivity, raise new requirements for the manufacturing equipment in terms of reliability, accuracy stability, automation, intelligence and availability of complete sets of equipment.

\subsubsection{Manufacturing equipment of aircraft structural parts}

With the continuous improvement of the performance of a new generation of aircraft, the aerospace monolithic component is becoming more and more complex, and its requirement of accuracy is increasingly high. More and more advanced structural materials such as titanium alloy and composites are used. The domestic 3-axis vertical machining center and gantry milling machine are developed to meet the strong processing needs at present. An important breakthrough, funded by major science and technology projects of the high-end CNC machine tools and basic manufacturing equipment, has been achieved, that the 5-axis high-torque $\mathrm{CNC}$ milling machine and the 5-axis high-speed $\mathrm{CNC}$ milling machine have been developed successively, but these machines should still be improved with regard to reliability and accuracy preservation. The development of 5-axis high-rigidity equipment meets the efficient machining requirements of the high-strength and high-precision large-scale flexible structural components in modern aircraft and the milling of titanium alloy parts. For instance, the powerful 5-axis CNC gantry machining center, 5 -axis horizontal boring and milling machining center, 5-axis vertical and horizontal conversion boring and milling center, still need to be developed to facilitate industrialization. The 5-axis high-speed large CNC machine tools and mirror milling equipment required in the manufacturing process of integral panels and framed aluminum alloy parts are still under development.

China has largely had the equipment of aircraft structural parts, but there is still a big gap in reliability and processing efficiency compared with the advanced foreign equipment. Over the past decade, the fault-free working hours of China's CNC machinery increased from $400-500 \mathrm{~h}$ to more than $1000 \mathrm{~h}$, but there is still a big gap from the $2000-3000 \mathrm{~h}$ fault-free working 
hours of CNC machinery from foreign countries. The machining efficiency can be only half that of the machinery of an advanced level in foreign countries, mainly because the analysis of the dynamic characteristics and the mechanical and electrical coupling characteristics of the multi-axis machine tool, and the forward design capacity are not very satisfactory. The solution is to strengthen industry-university-research collaboration.

\subsubsection{Manufacturing equipment of aircraft engine}

Most of the 5-axis milling NC machines of the blade profiles of aircraft engines are imported from abroad. Since the launch of the large aircraft special project and especially the special project of aeroengines and industrial gas turbines, the western countries have initiated a new round of embargoes against China. The stable and efficient processing of 5-axis blade CNC milling equipment with optimized blade profile processing software and convenient post-processing software for UG programming with online testing, blade defect repair, and other intelligent functions needs to be gradually developed. In the blade finishing process, the machine needs to have high acceleration linkage to process the small radius of curvature of the intake and exhaust side. It also needs to have 5-axis CNC grinding machine with process stabilization and appropriate dimensions to process various circular rabbets of compressor blades and circular mounting plates of guide wings.

The 5-axis precision boring and milling centers, 5-axis vertical machining centers, 5-axis lathe-milling machining centers, and precision $\mathrm{CNC}$ universal cylindrical grinders are indispensable for manufacturing casings, hubs, and other components. Domestic CNC equipment accounts for $25 \%$ of the existing cutting equipment whereas the imported accounts for $75 \%$. Domestic CNC equipment's ex-factory accuracy can satisfy the requirements of general-precision grade parts' finishing process, but the accuracy of the domestic equipment decreases rapidly and is not as satisfactory as that of the imported equipment.

More and more overall impeller disks and turbine disks in the engine structure present new challenges to the 5-axis and high-efficiency machining capabilities of CNC machines. Material increase manufacturing provides a novel efficient, lowcost way for this type of processing. The integration of material subtractive and additive technology and equipment will be an important direction of development in the future.

\subsubsection{Molding and processing equipment of composites}

With the large-scale and integrated development of composite structures in military aircraft and civil aircraft, the traditional hand-layup method can no longer meet the production requirements of large-scale and integrated components. There is no large-scale automated tape-laying equipment for composites in China, and automated tow placement technology and equipment for composites have been blocked by the developed countries. The equipment of automated tow placement should be equipped with functions of delivery, positioning, heating, rolling, and cutting for pre-preg tows. In addition, the further development of the large-scale autoclave with the diameter greater than $5 \mathrm{~m}$ and the length greater than $15 \mathrm{~m}$ is necessary to meet the research and production requirements of the large-scale overall structure of composite materials.

The amount of composite material used in the aircraft structure gradually increases. Unlike the cutting of metal materials, the processing of composites requires less splitting, better fiber cutting ability, and better surface quality. At present, the processing equipment of composite structural parts in China mainly relies on imports. The requirements for the processing and drilling equipment of the outer edge of large-size composite thin-walled parts and flexible fixture systems are considerable.

\subsection{Aerospace equipment}

According to a rough estimate, the number of the satellites that China needs to launch will be more than 200 by 2020. Classifying the satellites on the basis of the mass, satellites weighing less than 1 ton account for nearly $45 \%$, those weighing from 1 ton to 4 tons account for nearly $30 \%$, and those weighing more than 5 tons account for nearly $25 \%$. In near-earth orbit satellites, payloads weighing more than 5 tons are basically used for manned spaceflight projects such as cargo spaceship, space stations, and so on. Among the sun-synchronous orbit satellites, those weighing from 1 ton to 4 tons account for more than $50 \%$. In geostationary transfer orbit satellites and geosynchronous orbit satellites, the proportions of those of mass ranging from 1 ton to 4 tons and more than 5 tons are basically equivalent. However, with the development of China's new satellite platform in the future, it will be an inevitable trend to increase the quality of geosynchronous orbit satellites. From the discussion above, the payloads have a strong demand for the medium launch vehicle. The payloads of near-earth orbit satellites can be satisfied by the Long March 7 Launch Vehicle, while the sun-synchronous orbit satellites and the geosynchronous orbit satellites cannot be satisfied by current medium launch vehicles, and hence the development of medium launch vehicles is a key focus area [1].

The development of space equipment trends is as follows: (1) To meet the requirement of multipurpose, multifunctional, and long-life satellites, large-size, heavy-duty launch vehicles are essential. Because the satellite loads and the fuels to be carried to maintain and motivate the satellite become more and more, China should be able to manufacture the large-size, heavy-duty launch vehicles. (2) The space equipment tends to be of ultra-high precision. The structural shape of the aerospace equipment and the assembly of the parts are complex, requiring high dimensional accuracy, surface quality, and assembly precision and putting forward higher requirements on the machining precision of aerospace manufacturing technology. (3) On the other hand, the space equipment tends to be microsized. The 
small-size spacecraft has become the development trend of the international aerospace community. The design and manufacture of high-performance and small-size spacecraft have posed new requirements and challenges for the high-end aerospace manufacturing industry.

\subsubsection{Manufacturing technology and equipment of optical} sensor

Optical sensors are the core load of the satellites to achieve the observation of the objects in space. China's space optical sensor manufacturing aims at the international advanced manufacturing technology; hardware equipment and basic technology have made great strides, and the processing accuracy and efficiency have been greatly improved. However, compared with the future technological development trend of space optical sensors and the national demands of some major models, there is still a certain gap. Development of large-diameter plasma polishing equipment, ultra-large diameter and ultra-precision milling machines, ultra-large caliber intelligent polishing machines, and other high-end equipment to achieve large-diameter, ultra-large-diameter optical components of high efficiency and precision machining is the future direction.

\subsubsection{Manufacturing equipment of launch vehicle}

To achieve the goals of high performance, high reliability and low cost, launch vehicles have new demands for strength, stiffness, quality, and stability. Simultaneously, it puts forward higher requirements for the reliability and stability in processing control. Therefore, the production of launch vehicles is required to be "fast, efficient, highly reliable, digital, flexible, automatically assembled." Especially some required equipment is largesize thermoforming special equipment for the titanium alloy nozzle of heavy-duty launch vehicle engine, vacuum titanium superplastic forming/diffusion bonding and thermoforming line equipment, rocket structure with diameter of 3.35-10 m series efficient processing and precision welding equipment, high-precision and large-scale head sealing equipment of ultra-high strength steel with variable wall thickness, cylindrical powerful spinning CNC machine, high-precision and large-scale efficient laser arc hybrid welding and automated assembly equipment for ultra-high-strength steel housing, large-scale and high-precision laser arc composite welding and automated assembly equipment for hanging engine thick-walled saddle weld seam. In cutting, horizontal double spindle turning and milling combined machining CNC machine for large solid booster segmented metal shell, vertical milling and turning combined machining $\mathrm{CNC}$ machine for large diameter solid booster complex structure connecting of the ring shell and expansion segment of the shell, efficient vertical milling and turning combined machining equipment for large-diameter and thin-walled complex structure of aluminum skirts are needed. Large cylinder welding also requires large precision fixture tools and online processing equipment, the de- velopment of which is difficult and costly. Additive manufacturing equipment and material additive and subtractive integrated equipment can be innovative process and technology shortcuts that can avoid deformation during the processing, and should be vigorously developed.

\subsubsection{Aerospace composite materials manufacturing equipment}

Similar to the demand for composite materials in aeronautic equipment, composite components in aerospace equipment require complex shapes and high-precision composite material twining machines, large-size composite material fiber-spreading machines, and high-efficiency, high-speed, high-precision $\mathrm{CNC}$ composite cutting machine tools.

2.2.4 Precision / ultra-precision manufacturing equipment of aerospace complex structures

There are many parts with complex structures requiring many dimensional precisions and high shape precision that are difficult to process and deform easily, in aerospace inertial devices, position marker optical components, servo valves, and star sensors. Therefore, it is imperative to break through the bottleneck in aerospace precision/ultra-precision manufacturing and form a unique technology of aerospace precision/ultra-precision manufacturing inspection equipment. We should strive to achieve a shape processing precision no more than $0.5 \mu \mathrm{m}$ and a surface roughness no more than $80 \mathrm{~nm}$ in milling and grinding machines; and a shape processing precision no more than $0.1 \mu \mathrm{m}$ and a surface roughness no more than $4 \mathrm{~nm}$ in cutting machines.

\subsection{Rail transportation equipment}

In 2015, the output value of China's rail transportation equipment industry exceeded 400 billion yuan, ranking first in the world. China has built a batch of rail transit equipment manufacturing bases with world advanced level. The country has the capabilities of producing and serving for 2,000 high-powered locomotives, 8000 EMUs and railway passenger cars and urban rail vehicles, 60,000 various types of trucks, 500 large-scale maintenance machineries, 2,000 sets of overhaul locomotives, 5,000 EMU and various types of rail cars, and 70,000 various types of trucks. During the "13th Five-Year Plan" period, the demand for domestic rail transportation equipment will be strong and the growth rate will remain high. There are an increasing number of supporting products in the field of rail transit vehicles and ancillary equipment, and the hosts and market share grow year by year. With rise in labor costs along with fall in product prices, the demands of users become increasingly personalized and diverse. Diversified product configurations, increased design complexity, and reduced lead times have led to a speed-up of the entire value chain of manufacturing systems, resulting in products that meet the individual needs of users at lower costs. The developments in the rail transportation equipment manufacturing 
industry will be along the intelligent, automated, informatization, integration, high-speed, and green direction.

\subsubsection{Sheet forming equipment}

The sheet metal forming equipment includes high-power $\mathrm{CNC}$ forming machines for bending various parts of locomotives and urban rail products, $\mathrm{CNC}$ pressure forming machines, $\mathrm{CNC}$ bending machines, laser and plasma cutting machines for sheet cutting, high-power hydraulic machines for surface parts forming, and NC step machine for punching sheet metal parts.

\subsubsection{Machine tool for train body}

High-speed gantry machining centers and large vertical legislation processing centers and highly flexible production lines consisting of $\mathrm{CNC}$ boring and milling machines for large car body processing, and double turret vertical $\mathrm{CNC}$ turning centers and multi-axis turning and milling centers and high-precision grinding centers for wheels and axles processing are needed.

\subsubsection{Machine tool for small parts}

Gear transmission systems and forged steel brake discs have higher demands on machining equipment. Common hobbing machine production has low efficiency and poor machining accuracy, making it difficult to meet the requirements of mass production, and impacting correct engagement and service life. Six-axis CNC hobbing machines are commonly used in foreign countries, whereas domestic machines cannot guarantee the precision of rolling stock products. Grinding machines are needed with a modulus no more than $40 \mathrm{~mm}$, a tooth width larger than $580 \mathrm{~mm}$, and an accuracy of $3-4^{\circ}$. The forged steel brake disc with heat sink is one of the key components in the high-speed EMU basic braking system, and is characterized by a large diameter plate, a high heat sink, and small thickness. Forgings are made of $30 \mathrm{CrNiMoA}$ and weigh approximately $100 \mathrm{~kg}$. As there are up to 24 heat sinks evenly distributed on the disk surface, the heat sinks are not easy to machine and thus need to be forged.

\subsubsection{Sheet automatic welding}

In the large plate welding process, the laser (plasma)-arc hybrid welding equipment, friction stir welding equipment, automatic polishing and grinding machines and all kinds of automatic welding equipment are needed. The laser welding technology database of medium- and thick-plate metal materials is established to realize the engineering application of laser welding of large parts of rolling stock and the application of the new friction stir welding technology of thick plates (such as coupler plate and bolster).

\subsection{Ocean engineering equipment}

With the rapid development of offshore oil and gas engi- neering equipment, the exploration of deep water oil and gas in South China Sea etc., has become China's area of key focus in its mid-to-long-term energy development plan. In recent years, China has made a remarkable progress in the field of research and manufacturing of ocean engineering equipment. Significant breakthrough happens in key technologies and conceptual equipment, such as large-scale floating liquefied natural gas production systems, large-scale floating drilling production storage and offloading systems, and large-scale semi-submersible drilling and production platforms. In addition, large-tonnage column crane and large low-voltage towing machine have already started to be built. Besides, it is also necessary to develop the seabed mineral resources exploration equipment, build and improve a series of technologies and equipment of multiple deepsea resources, give priority to the pre-mining system design and deep-sea test with great business prospect, and build world-class integrated test bases.

Performance requirements of ocean engineering equipment are increasingly demanding. To overcome the difficulties of many super-large complex curved surfaces, narrow and severe manufacturing environment, and extremely high demands for precision of key equipment and facilities, high precision, high quality, high efficiency, and low cost of the manufacturing equipment becomes accordingly increasingly important.

\subsubsection{Machining equipment for high-precision platform plate}

Large equipment with heavy weight, big volume, and high requirement is installed on the offshore platform, which requires a stable foundation. A 1000-ton platform crane needs a highprecision round base with a diameter of $10 \mathrm{~m}$. Drilling moonpool needs a $60-\mathrm{m}$ guide with the precision of $0.5 \mathrm{~mm}$. A machine tool for high-precision processing of large and super-large planes in a narrow space of the platform is needed. The offshore platform demands a single product with varieties of sizes and thicknesses, necessitating a highly flexible machine tool that can precisely make sheet steel with different shapes, thicknesses, and materials.

\subsubsection{Machining equipment for complex surface of propeller}

During the manufacturing of large propellers, processing of end planes, axle holes, and blades is the key steps. All sorts of $\mathrm{CNC}$ machine tools are used, including a high-precision vertical lathe for the diameter and depth processing of the hub, a multi-axis $\mathrm{CNC}$ milling machine for large propeller with a span of more than $11 \mathrm{~m}$, an automatic CNC grinding machine for the blade surface, leading edge and trailing edge, high-end millinggrinding machine tool to improve the manufacturing capability.

\subsubsection{Machining equipment for deep hole}

The stern and rudder axle hole is of a deep hole structure bearing heavy load at operation. Therefore, it is necessary to 
ensure high standards for concentricity and roughness during the processing. A relatively large error could have a serious impact on the stern axle installation and the safety and efficiency of the ship operation. The deep hole is up to 700-1200 $\mathrm{mm}$ in diameter and more than $2000 \mathrm{~mm}$ in depth and is usually processed outdoors. With the existing boring machine, it is hard to ensure a high precision because of structure deflection and the environment temperature. As a result, it is necessary to develop a deep hole boring machine that is suitable for outdoor machining environment to perform the following functions: on-machine high-precision measurement, automatic error compensation, and overcoming the influence of guide rod deformation, tool wear and temperature changes on manufacturing accuracy. Besides, a medium-speed diesel engine has a large number of complex oil circuits, with a maximum aspect ratio more than 30 and pore wall roughness less than 1.6. High-precision deep-hole drilling equipment is necessary in this case.

\subsubsection{Machining equipment for main engine}

A low-speed marine diesel engine consists of three parts: cylinder block, frame, and mount, whereas a medium-speed diesel engine consists of independent parts. To accomplish the process of these parts, $\mathrm{CNC}$ gantry boring-milling machine with a table of size more than $4 \mathrm{~m} \times 12 \mathrm{~m}$ and a CNC drilling machine (spindle diameter no less than $200 \mathrm{~mm}, \mathrm{X}$-axis travel no less than 10 $\mathrm{m})$ are required. As for the connecting rod and the piston rod, a series of machinery are essential, including $\mathrm{CNC}$ gantry boringmilling machines, $\mathrm{CNC}$ horizontal boring-milling machine centers, $\mathrm{CNC}$ horizonal turning, deep-hole drilling machines, and precision grinding machines.

\subsubsection{D printing equipment for repairing}

Marine equipment is away from manufacturing enterprise at runtime and hence it is difficult to find replacements when the parts fail or are damaged. A 3D printer provides the capability of on-site repair. Hence, 3D printing equipment should be installed according to the group classification and storage space of wearing parts.

\subsection{Intelligent manufacturing equipment}

With the development of high-end manufacturing equipment technology, sensors and intelligent process control software need to be developed to improve the machining efficiency and maintain a stable quality of processing. Moreover, with manufacturing big data, by using analysis of machining examples and autonomous learning, the process and design of manufacturing equipment can be optimized. Consequently, building big data platforms, carrying out automatic machine learning, and using virtual reality technologies are needed. Then, the equipment manufacturing industry could enter the intelligent level, which would further improve the efficiency of equipment and production systems.

\section{Suggestions for the development of high-end manufacturing equipment}

History demonstrates that a strong manufacturing capability is the foundation for achieving a high-level and strong ability for high-end equipment, dominant factor of international influence, and key support for the implementation of the Belt and Road Initiative. If the West keeps blockading on China's high-end manufacturing equipment, it will seriously limit the rapid development of large aircrafts, satellites, high-speed trains, and marine equipment in China. Since the major national science and technology project of high-end $\mathrm{CNC}$ machine tools and basic manufacturing equipment was carried out eight years ago, the high-end $\mathrm{CNC}$ system bottleneck has been broken through, filling some of the gaps of high-end machine tools and bringing the research of machine tools to the field of aeronautic and aerospace manufacturing equipment. However, the wide application of domestic equipment is still difficult because of the institutional constraints and the need for improving through feedback. To enhance military manufacturing capability and maintain information security of the defense industries, suggestions are put forward as follows:

(1) The most serious problem associated with imported software for CNC machining is that this kind of software often has information back doors and is monitored; so is the software applied used in military products and equipment. To avoid information leakage, independent software (such as CAD, CAE, or some other software) should be developed in China. The development of such basic software requires years of technology accumulation, but it would be much easier to develop professional software based on common software systems. In this way, the product design in China can be isolated from imported software and the information security can be guaranteed.

(2) Push the purchase plan of military products. To protect national defense, every insecure factor should have a veto power over the purchase of military products. Furthermore, China's equipment manufacturing enterprises and the purchase of domestic equipment should be supported.

(3) Break institutional constraints and encourage machine tool enterprises to enter the field of aeronautic and aerospace manufacturing. With these strategies, the development opportunities can be provided for the machine tool industry, and the problems of improving the production capability of aeronautic and aerospace industry and developing access to the machine tool industry can be solved.

(4) Continue to support the major science and technology project of high-end $\mathrm{CNC}$ machine tools and basic manufacturing equipment. Concretely, China should support the achievements of the project to enable its industrialization and upgrade the 
industry to facilitate intelligent manufacturing, to meet the demands of the rapidly developing aeronautics and astronautics field. Adopting this approach will allow China's CNC equipment to be near the top of international rankings and promote the "Made in China 2025" plan of China as well.

(5) Start the major science and technology project for intelligent manufacturing as soon as possible. Thus, the major science and technology project for high-end CNC machine tools and basic manufacturing equipment can be extended to the stage of intelligent manufacturing.

\section{References}

[1] Yang B H, Chi T H. Thinking for the development of satellite remote sensing application industry [J]. High-Technology \& Industrialization, 2010, 6(12): 26-29.Chinese 